\title{
A Fast Drying Machine Based On Semiconductor Heat Pump
}

\author{
Fengzhe.Wang ${ }^{1 *}$ \\ ${ }^{1}$ Department of energy and power engineering, Wuhan University of Technology, Wuhan, Hubei, 430000, China
}

\begin{abstract}
The new latent heat recovery type semiconductor heat pump ground fast dryer is a kind of integrated semiconductor refrigeration module on the traditional dryer. We simulated the process of the wind being heated by the hot end of the semiconductor refrigeration module under different working conditions through ANSYS software, so as to obtain the best solution for the semiconductor refrigeration module. Using the principles of drying and air condensation, we calculated the influence of the drying rate, drying time, and the condensation effect of the cold end when the air at different hot end inlet wind speeds, ambient temperature and moisture content blows the same amount of moisture on the ground. Through energy-saving analysis, our design saves about $60 \%$ energy compared with traditional electric heating style hair dryers. In the case of the same input power, the outlet air temperature of the semiconductor heat pump ground rapid dryer is significantly higher than that of the electric heating hair dryer. Therefore, when the ground is dry with the same amount of moisture, the use of the new semiconductor heat pump ground rapid dryer can significantly shorten drying time, the best drying time shortening rate is as high as $70 \%$.
\end{abstract}

\section{Introduction}

In our lives, we often see a lot of residual water on the floor that has just been cleaned[1]. On the one hand, this slippery floor may cause personal injury to customers or passengers, and on the other hand, it may cause personal injury[2]. Therefore, a quick-drying device after mopping is essential. In addition, in hotels and even at home, hair dryers are also used to quickly dry carpets on the ground. However, because the blown air is at a normal temperature, the temperature is relatively low, which seriously affects the drying rate and drying efficiency. At present, some parts of the domestic market use electric heating hair dryers. While increasing the outlet temperature, they only rely on electric heating to consume more electricity[3-4]. However, in other areas of drying technology, an energysaving technology that uses latent heat to optimize the drying effect has emerged. For the sake of energy saving, the small size, simple structure and easy to install hot air dryer have been developed[5-6]. On the one hand, it speeds up the evaporation rate of water and greatly shortens the time required for drying. On the other hand, it improves the discomfort caused by wind blowing on pedestrians[7].

\section{Materials and Methods}

\subsection{Drying calculation of the hot end}

The blow dryer blows away the moisture on the ground, and the ground surface is in a wet state, and its moisture is unbound moisture, so the process of drying the ground by the blow dryer is regarded as a constant-speed drying stage. The dry-ing rate of the constant-rate drying stage is only related to the state of the air, that is, only related to the temperature, flow rate and moisture content of the air[8].

In the constant-speed drying stage, the surface temperature of the material is equal to the wet bulb temperature of the air, and the heat transfer rate of the air to the material is equal to the rate at which the moisture vaporizes and takes away the latent heat[9-11]. The drying rate (also called water vaporization rate) NA in the constant-rate stage, that is, the amount of water vaporized per unit time per unit area, equal to a constant, and its calculation formula is the mass transfer or heat transfer rate formula:

$$
N_{A}=k_{H}\left(H_{\omega}-H\right)=\frac{\alpha}{r_{\omega}}\left(t-t_{\omega}\right)
$$

Among them, $\alpha$-heating coefficient, unit $\mathrm{kW} / \mathrm{m} 2 \cdot{ }^{\circ} \mathrm{C}$, when air flows parallel to the surface of the material, $\alpha=0.0143 G^{0.8}$;

$r_{w}$ - The latent heat of vaporization at wet bulb temperature, in $\mathrm{kJ} / \mathrm{kg}$;

$t$ - The temperature of the air flow, in ${ }^{\circ} \mathrm{C}$;

$t_{w}$ - Wet bulb temperature of air, in ${ }^{\circ} \mathrm{C}$.

The drying time in the constant speed stage is:

$$
\tau=\frac{G_{c}}{A} \cdot \frac{X}{N_{A}}
$$

Among them, $\mathrm{Gc}$ - the quality of dry materials, which is regarded as unit mass material $(\mathrm{Gc}=1 \mathrm{~kg}$ material);

$A$ - The area of the gas-solid contact interface, which is regarded as a unit area $(\mathrm{A}=1 \mathrm{~m} 2)$;

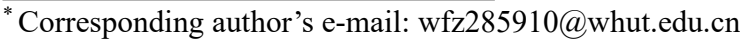


$X$-water content, where $\mathrm{X}=0.1 \mathrm{~kg}$ water $/ \mathrm{kg}$ dry material in unit area;

$N_{A}$ - the amount of water vaporized per unit time per unit area, unit $\mathrm{kg} /(\mathrm{m} 2 \bullet \mathrm{s})$.

\subsection{Condensation calculation of the cold end}

During the condensation process, the actual cold quantity Qa absorbed by the moist air to be treated is composed of the sensible heat Qx of the moist air and the latent heat Qq of condensation:

$$
Q_{a}=Q_{x}+Q_{q}=m c \Delta T+m_{w} \gamma=m_{d a} \Delta h+m_{w} h_{0}
$$

Among them, $\mathrm{m}_{\mathrm{da}}$ - dry air quality, in $\mathrm{kg}$;

$\Delta h$ - The enthalpy difference between the moist air in the initial and final state, in $\mathrm{kJ} / \mathrm{kg}$ dry air;

$h_{0}$ - The enthalpy of condensate in the final state, in $\mathrm{kJ} / \mathrm{kg}$ dry air;

$m_{w}$ - the quality of condensed water, that is, the amount of condensation of the semiconductor refrigeration device, in $\mathrm{kg}$.

\subsection{Overall design and principle}

The outlet air of the hair dryer passes through the hot end of the semiconductor refrigeration device, which is heated and heated and blown to the damp ground to absorb moisture on the ground, while the cold end of the semiconductor refrigeration device is exposed to the environment, and the air in the environment is The cold end of the semiconductor device condenses into droplets. On the one hand, the cold end of the semiconductor refrigeration device absorbs the latent heat of condensation of the air and transfers it to the hot end of the semiconductor refrigeration device. The ambient air plays a role of local dehumidification, which reduces the initial moisture content of the air passing through the hot end of the semiconductor refrigeration device, which is more conducive to the evaporation of ground moisture. Therefore, it is necessary to ensure both the heating effect of the hot end and the condensation effect of the cold end.

\subsection{Drying calculation of the hot end}

According to the above design ideas, ANSYS software is used to create a semiconductor refrigeration model to simulate the temperature of the outlet air and the temperature of the cold end heat conduction fin when the outlet air of the dryer flows through the hot end of the semiconductor refrigeration model.

The input power of the hair dryer is $1000 \mathrm{~W}$, and the three speeds are adjustable. Using the direct modeling function in the ANSYS software, the outlet of the dryer is simplified into a rectangle with a width of $380 \mathrm{~mm}$ and a height of $76 \mathrm{~mm}$, which is the hot end of the semiconductor refrigeration model. import. The rated voltage of the cooling plate is $12 \mathrm{~V}$, and the current flowing through the semiconductor cooling plate is set to $9 \mathrm{~A}$ in the simulation. The materials of the hot-end radiator and the cold-end conduction fins are made of extruded aluminum, with a thermal conductivity of $205 \mathrm{~W} / \mathrm{mK}$. The size of the cold- end conduction fins is $45^{*} 40 * 23 \mathrm{~mm}$, which corresponds to the cooling fins one to one. In the simulation, the average wind speed of the first gear is $17 \mathrm{~m} / \mathrm{s}$ as the inlet wind speed of the semiconductor refrigeration device, and the initial ambient temperature is assumed to be $20^{\circ} \mathrm{C}$.

\subsubsection{Determination of the number of semiconductor} refrigeration fins. With reference to the radiator structure on the market, the width of the hot-end radiator is initially set to be $380 \mathrm{~mm}$, which is consistent with the outlet width of the blow dryer. The rib height is set to $55 \mathrm{~mm}$, the fin thickness is $1 \mathrm{~mm}$, and the ratio of the number of fins to the width is 0.2 . That is, the number of fins is 76 . The evenly-arranged 6 and 9 semiconductor refrigeration fins have a temperature rise of $5 \sim 10^{\circ} \mathrm{C}$ on the wind near the ground at the bottom of the dryer.

\subsubsection{Determination of the number of fins on the hot} end radiator. The density of the hot-end radiator affects the heat dissipation of the cooling fins. Too many fins will cause excessive wind resistance, resulting in a serious loss of wind speed and affecting the drying effect. If the number of fins is too low, the heat dissipation of the semiconductor refrigeration fins will not be timely, and the effect of the cold end of the semiconductor refrigeration model will be deteriorated. The cold end temperature will be too high and the condensation function will be lost. Therefore, it is necessary to select the appropriate number of fins. According to our calculations, 76 fins on the hotend radiator have the best effect.

\subsection{Design of air duct}

Generally speaking, in the process of ground drying, the main factors affecting its drying rate are wind temperature, wind speed, wind volume, and moisture content. In the final analysis, the dryness of the ground is that the wind parallel to the ground takes away the moisture on the surface of the floor. Therefore, the influence of wind speed and air volume on drying is second only to relative humidity, and it can be realized at a relatively low cost. Therefore, a good drying system should pay attention to the design of the air duct and flow rate. The outlet air duct with a certain angle between the design and the level ground is designed to reasonably distribute the outlet wind speed and air temperature of the dryer to achieve the purpose of improving the drying effect. We found that when a $200 \mathrm{~mm}$ long air duct at an angle of $5^{\circ}$ with the horizontal ground is arranged behind the hot-end radiator, the drying rate of the dryer is the fastest and the effect is the best. Through the comparison of mass flow, when the air outlet flow channel of the dryer is arranged in this working condition, the mass flow of the two planes is higher than the other working conditions, which is beneficial to increase the drying rate. Through the area integral comparison of velocity and temperature, it can be seen that within the range of this experimental simulation, the average velocity and average temperature in the plane increase with the angle, and the mass flow in this working condition is the largest. However, if the angle is too large, 
the hot air coverage distance will be shortened sharply, and it will also cause flow loss.

\section{Results and Discussion}

\subsection{Energy saving efficiency}

In some literatures, electric heating is used to heat up the wind blowing to the ground, thereby increasing the drying rate. When the semiconductor refrigeration device is used for heating purposes, it is also called a "semiconductor heat pump", which moves heat from the low temperature end to the high temperature end.

Heating capacity of semiconductor refrigeration module:

$$
Q_{h}=P+Q_{c}
$$

Heating coefficient:

$$
\varepsilon_{h}=\frac{Q_{h}}{P}=\frac{Q_{c}}{P}+1
$$

Among them, Qc and Qh are respectively the cooling capacity of the cold end and the heating capacity of the hot end per unit time of the cold and hot ends, $\mathrm{P}$ is the electric power provided by the outside to the cooling plate, and $\mathrm{P}$ fan is the electric power provided by the outside to the fan. We found that in the case of an ambient temperature of $20^{\circ} \mathrm{C}$ and 9 semiconductor cooling chips (working current 9A), within the studied wind speed range, the energy saving efficiency decreases with the increase of the hot end inlet wind speed, and the energy saving efficiency is $72.53 \%$. When the inlet wind speed of the hot end is set at $17 \mathrm{~m} / \mathrm{s}$ and 9 semiconductor cooling fins (working current 9A), within the temperature range studied, the energysaving efficiency increases with the increase of the ambient temperature, and the energy-saving efficiency is $69.50 \%$.

\subsection{Fast drying characteristics}

Compared with traditional dryers, semiconductor heat pump ground fast dryers need to provide additional power supply for semiconductor refrigeration chips, but use the same amount of power to process the same air volume in the form of electric heating, and its temperature rise effect is not as good as semiconductor heat pumps Ground fast dryer. Therefore, compared with the traditional electric heating dryer, the semiconductor heat pump ground fast dryer can significantly shorten the drying time.

\subsection{Analysis of key technologies}

Compared with conventional semiconductor heat pump dryers, the highlight of this new type of semiconductor heat pump ground fast dryer is its realization of high COP. Semiconductor heat pump dryers under different COPs, when drying the same amount of moisture, the researched $\mathrm{COP}=4.5$ new type semiconductor heat pump ground fast dryer requires the shortest time, and during this working time, its cold end condensation effect Optimal, thus making full use of the latent heat recovery capacity of the cold end.

In our research, the realization of high COP is mainly based on the following two points:

The first point is the reasonable arrangement of the hot-end radiator and cold-end conduction fins. Through calculations by ANSYS Icepak software, the structure of the hot-end radiator (contact area, number of fins) and the air volume of the hot-end are reasonably matched so that the air volume of the hot-end can achieve a considerable temperature rise after flowing through the hot-end radiator. , It can also fully take away the heat of the hot end; the cold end of the cold end adopts a distributed arrangement, so that the heat dissipation area of the cold end can ensure the sufficient condensation effect of the cold end. If it is an integrated arrangement, the heat dissipation area of the cold end is too large The condensing effect is greatly reduced, so that the heat absorbed by the cold end and transferred to the hot end is reduced, resulting in a decrease in COP.

The second point is that under different working conditions, focusing on the best matching air volume of the cold end is another key factor. For a given working condition, the corresponding cold end temperature is constant. At this time, if the ventilation volume of the cold end is too large, but the cold end is not enough to handle the latent heat of these air volumes, the amount of air below the dew point is relatively small. Most of the absorbed heat is sensible heat, so the heat transferred to the hot end will also be reduced; while the ventilation of the cold end is too small, it wastes its ability to deal with the latent heat of the air, so that the absorbed heat is not maximized; therefore, it should be reasonable Matching the ventilation volume of the cold end makes it fully absorb the latent heat of condensation of the air.

\section{Conclusions}

Our design integrates the semiconductor refrigeration device into the traditional dryer application, so that the wind at room temperature is heated and blown to the ground, greatly increasing the drying rate, significantly shortening the drying time, and extending the service life of the semiconductor heat pump ground fast dryer. Compared with the electric heating dryer, the drying effect is remarkable, and the drying time of the designed product is shortened by $40 \sim 70 \%$. Our semiconductor heat pump ground fast dryer utilizes its cold end to absorb the latent heat of condensation of moist air and transfer the heat to the hot end, thereby reducing the amount of electricity provided by the outside world, thus achieving the purpose of energy saving. In addition, our design uses the cold end to condense the humid air, which makes the air around the new type of semiconductor heat pump ground fast dryer have a lower moisture content, thereby reducing the initial moisture content of the air passing through the hot end of the semiconductor refrigeration device, which is more beneficial Dryness of ground moisture. Our research has found that the drying effect of the new type of semiconductor heat pump ground fast dryer under low wind speed can reach the drying effect of the traditional electric heating dryer under high wind speed. Therefore, 
the fan of the traditional dryer can be replaced with a fan of lower power, thereby further reducing energy consumption.

\section{References}

1. Wang Jingwei. Research on the performance of thermoelectric heat pump drying device [D]. Hunan University, 2004.

2. Yang Xiurong. Research on electronic temperature control and dehumidification technology in small environment [D]. Hefei University of Technology, 2012.

3. Guo Zongkun, Yao Lei, He Kai, et al. Development of dehumidification device based on semiconductor refrigeration technology $[\mathrm{J}]$. High-power converter technology, 2016(6):71-75.

4. Xie Ling. Development and research of small thermoelectric refrigeration (dehumidifier) [D]. Hunan University, 2007.

5. Wang Yongkang, Zhang Yifang. ANSYS Icepak advanced application navigation case[M]. China Water Resources and Hydropower Press, 2016.

6. Wang Xinlan. Analysis of the variable frequency twostage compression system of the heat pump dryer [J]. Agricultural Technology and Equipment, 2020(09): 122-124.

7. Su Yinghe, Liu Mingjun, Chen Tao. A sludge drying and dehumidification system based on an absorption heat pump $[\mathrm{J}]$. Electromechanical Information, 2020(19): 76-80.

8. Li Guojian, Cui Yunhan, Wu Shuang, Duan Pengsheng, Ye Dapeng. Fluent modeling and simulation analysis of flow field in heat pump drying room[J]. Journal of Fujian Agriculture and Forestry University (Natural Science Edition), 2020, 49(03): $425-432$.

9. Li Huan, Zhang Pengfei, Dou Weibiao. Application of heat pump-electric heating combined dryer in food drying[J]. Light Industry Science and Technology, 2020, 36(05): 6-7.

10. Liu Jinlong, Zheng Shiqing, Cao Shaoji, Zhang Yinghong, Zheng Linwei. Experimental research on synthetic laponite heat pump drying $[\mathrm{J}]$. China Nonmetallic Mineral Industry Guide, 2019(03): 17-19.

11. Liu Zhongshang, Zhang Xiaodan, Gao Bo. Research on scroll compressors used in the field of hightemperature drying heat pumps $[\mathrm{J}]$. Refrigeration and Air Conditioning, 2019, 19(07): 84-87. 